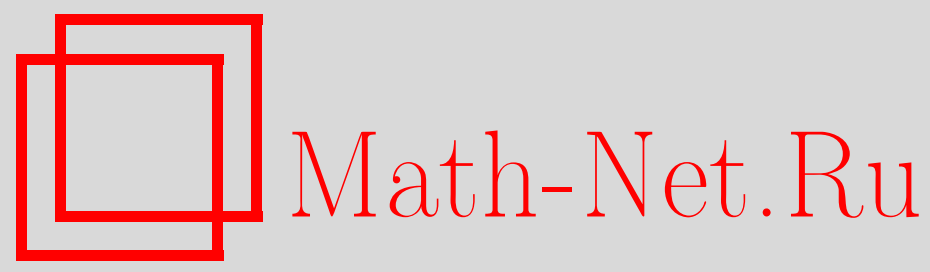

П. Дундар, А. Айтак, Выражения для целостности тотальных графов через некоторые характеристики графов, $M a-$ тем. заметки, 2004, том 76, выпуск 5, 714-722

DOI: https://doi.org/10.4213/mzm141

Использование Общероссийского математического портала Math-Net.Ru подразумевает, что вы прочитали и согласны с пользовательским соглашением http://www . mathnet.ru/rus/agreement

Параметры загрузки:

IP : 54.198 .55 .26

26 апреля 2023 г., 04:35:53

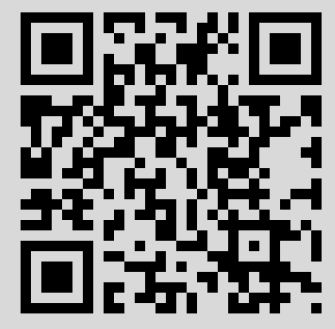




\title{
ВЫРАЖЕНИЯ ДЛЯ ЦЕЛОСТНОСТИ ТОТАЛЬНЫХ ГРАФОВ ЧЕРЕЗ НЕКОТОРЫЕ ХАРАКТЕРИСТИКИ ГРАФОВ
}

\author{
П. Дундар, А. Айтак
}

Коммуникационные сети должны обладать высоким уровнем надежности. Вообще говоря, обрывы соединений, отключение узлов сети, ошибки программного обеспечения или аппаратные сбои, равно как и сбои при передаче данных, могут привести к долговременным отказам системы. Коммуникационные же сети требуют большей устойчивости, или меньшей уязвимости. Уязвимость коммуникационной сети измеряет способность сети сопротивляться разрушению в результате отказа некоторых станций или звеньев связи. Для описания устойчивости коммуникационной сети в терминах графа $G$, моделирующего сеть, используются разнообразные характеристики графа, такие как связность, целостность и прочность. Рассмотрим два графа с одинаковой связностью, но с различной величиной наибольших компонент связности, возникающих при удалении ребер. Ясно, что эти два графа должны характеризоваться различной устойчивостью. Как измерить это отличие? В результате мы приходим к понятию целостности, отличному от связности. Большой класс графов образуют тотальные графы. В настоящей статье мы описываем целостность тотальных графов в терминах некоторых характеристик графов.

Библиографоия: 23 названия.

1. Введение. Отказ соединений и узлов коммуникационной сети может привести к потере ею эффективности. Поэтому коммуникационные сети должны делаться максимально устойчивыми не только к разрушениям, но и к возможным перестройкам. При анализе уязвимости коммуникационной сети при ее нарушениях следует иметь ввиду следующие две величины:

1) размер максимальной групшы узлов, сохранившей связь между любьми двумя узлами;

2) количество неработающих элементов.

Для описания устойчивости коммуникационной сети, смоделированной на графе, применяется цельй ряд параметров, используемых в теории графов, среди которых связность, целостность, грубость, стойкость и коэффициент связности.

Граф $G$ представляет собой пару $G=(V(G), E(G))$, где $V(G)$ - это множество вершин графа $G$, а $E(G)$ - его множество ребер. Число вершин и число ребер графа $G$ обозначаются, соответственно, через $|V(G)|,|E(G)|$. Эффективность графа, моделирующего топологию сети [1], падает при разрушении (по любой причине) некоторых его вершин или ребер.

(Вершинная) связность графа $G$ равна минимальному числу вершин, удаление которых приводит к несвязному или тривиальному графу; она обозначается через $k(G)[2]$. 
Реберная связность графа $G$ равна минимальному числу ребер, удаление которых приводит к несвязному или тривиальному графу; она обозначается через $k_{1}(G)$ [2]. Связность графа равна минимальному числу вершин, удаление которых разбивает его на две или большее количество компонент связности. Однако для двух графов с одинаковой (вершинной или реберной) связностью и одинаковьм числом вершин число вершин в максимальной компоненте, полученной в результате такого распадения, может оказаться различным. Это означает, что связность не может полностью характеризовать устойчивость. Помимо связности в теории графов для характеризации устойчивости используются такие величины как целостность, окрестностно-целостная грубость, реберная целостность, реберная окрестностная целостность и прочность [3]-[15].

В начале этой статьи мы используем в качестве меры устойчивости графа целостность, а затем описываем некоторые параметры тотальных графов для графов из некоторых классов и вводим понятие целостности тотальных графов.

Целостность графа $G$ определяется равенством

$$
I(G)=\min _{S \subseteq V(G)}\{|S|+m(G-S)\},
$$

где $V(G)$ - множество вершин графа $G$, а $m(G-S)$ - максимальное количество вершин в компоненте связности графа $G-S$. Это понятие было введено Багга, Барфутом, Энтрингером и Свортом [16]-[18]. I-множеством в $G$ назьвается произвольное (строгое) подмножество $S$ в $V(G)$, для которого $I(G)=S+m(T(G)-S)$ [19].

Для графа $G$ порядка $p$ справедливы неравенства $1 \leqslant I(G) \leqslant p$, и если $H$-подграф в $G$, то $I(H) \leqslant I(G)$.

Если $G=\bigcup_{i=1, \ldots, n} G_{i}$, то

$$
\max _{i} I\left(G_{i}\right) \leqslant I(G) \leqslant \sum_{i=1}^{n} I\left(G_{i}\right)-n+1 .
$$

Для любых графов $G$ и $H$ справедливо равенство

$$
I(G+H)=\min \{I(G)+|H|, I(H)+|G|)\} .
$$

Несмотря на то, что задача вычисления целостности графов $N P$-полна, для больших классов графов сложность можно найти явно [20]-[22].

Вершины и ребра графа назьваются его әлементами. Два элемента графа называются соседями, если они инцидентны друг другу или примыкают друг к другу. Тотальным графом $T(G)$ графа $G=(V(G), E(G))$ называется граф с множеством вершин $V(G) \cup E(G)$, причем две вершины в тотальном графе соединены ребром в том и только в том случае, если соответствующие им элементы в $G$ являются соседними. Легко видеть, что $T(G)$ содержит в качестве индуцированных как подграф $G$, так и реберньй подграф $L(G)$. Важной особенностью тотального графа является его максимальность среди всех графов, образованных с помошью отношения примыкания в графе [23].
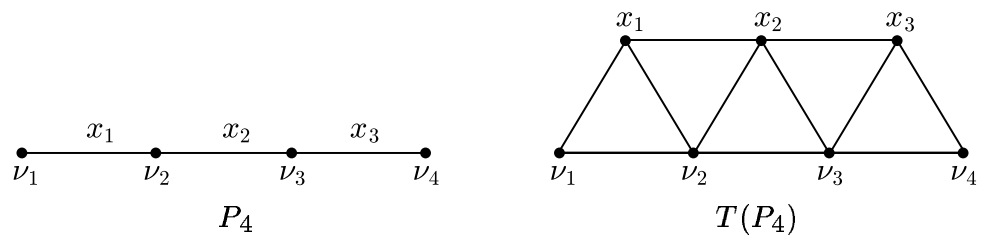

Рис. 1. Построение тотального граффа 
2. Основные результаты о целостности. Начнем со значений целостности для графов разных типов [16].

РЕзУльтат 2.1. Целостность полного графа $K_{n}$ равна $I\left(K_{n}\right)=n$.

РезУльТАТ 2.2. Целостность звезды $K_{1, n}$ равна $I\left(K_{1, n}\right)=2$.

Результат 2.3. Целостность цепочки $P_{n}$ равна $I\left(P_{n}\right)=\lceil 2 \sqrt{(n+1)}\rceil-2$.

РезУльтат 2.4. Целостность цикла $C_{n}$ равна $I\left(C_{n}\right)=\lceil 2 \sqrt{n}\rceil-1$.

РезУльтат 2.5. Целостность сетки $P_{m} \times P_{n}$ при $n \geqslant 2, n=r^{2}+k$ и $0<k<2 r$ равна

$$
\begin{aligned}
& I\left(P_{2} \times P_{n}\right)= \begin{cases}2 I\left(P_{n}\right)-1, & 0 \leqslant k \leqslant r / 2 \text { или } r \leqslant k<3 r / 2, \\
2 I\left(P_{n}\right) & \text { в противном случае, }\end{cases} \\
& I\left(P_{3} \times P_{n}\right) \leqslant 2(\lceil\sqrt{3 n+1}\rceil-1), \quad \text { если } n \text { нечетно, } \\
& I\left(P_{3} \times P_{n}\right) \geqslant 2\lceil\sqrt{3 n}\rceil-1, \quad \text { если } n \text { четно. }
\end{aligned}
$$

РезУльтат 2.6. Целостность гиперкуба $Q_{d}$ равна $I\left(Q_{d}\right)=2^{d-1}+1$, где $d$-размерность гиперкуба.

РезУльтат 2.7. Для любого графа $G$ имеют место неравенства $\delta(G)+1 \leqslant I(G) \leqslant$ $\alpha(G)+1$, и если $\alpha(G)=\delta(G)$, то $I(G)=\alpha(G)+1$, где через $\delta(G)$ обозначена минимальная степень вершины, а $\alpha(G)$ обозначает число покрытия графа [19]; $\Delta(G)$ обозначает максимальную степень вершины в $G$.

РЕзУльтат 2.8. В любом неполном графе $G$ всякое его $I$-множество является разделяющим, и поэтому мощность такого множества не меньше, чем $k(G)[19]$.

3. Некоторые результаты о характеристиках тотальных графов. В этом разделе мы определяем некоторые характеристики графов и находим их значения для тотальных графов.

ОПРЕДЕЛЕНИЕ 3.1. Подмножество $S$ в $V(G)$ такое, что, по крайней мере, один из конщов каждого ребра в $G$ лежит в $S$, называется покрывающим множеством. Число вершин в минимальном покрывающем множестве графа $G$ называется числом покрытия этого графа и обозначается через $\alpha(G)$.

ОПРЕДЕЛЕНИЕ 3.2. Подмножество $S$ в $V(G)$ назьвается независимым, если никакие две из его вершин не примыкают друг к другу. Число вершин в максимальном независимом множестве в $G$ называется индексом независимости этого графа и обозначается через $\beta(G)$. Порядок $n$ графа $G$ определяется равенством $\alpha(G)+\beta(G)=n$.

ОПРЕДЕЛЕНИЕ 3.3. Вериинно доминирующее множество в графе $G$ - это такое множество $S$ его вершин, что всякая вершина в $G$ либо лежит в $S$, либо примькает к вершине из $S$. Минимальное количество вершин в вершинно доминирующем множестве графа $G$ назьвается индексом вершинного доминирования этого графа и обозначается через $\sigma(G)$. Для любого графа $G$ справедливо неравенство $\sigma(G) \leqslant \beta(G)[2]$.

Через $\delta(G)$ мы обозначаем минимальную степень вершины в $G$. В этой работе наименьшее целое число большее $x$, обозначается через $\lceil x\rceil$, наибольшее целое число меньшее $x$, обозначается через $\lfloor x\rfloor$, а абсолютная величина числа $x$ обозначается через $|x|$. 
Tеорема 3.1. Пусть $P_{n}-$ чепочка с $n$ вериинами. Тогда

$$
\begin{gathered}
k\left(T\left(P_{n}\right)\right)=2, \quad \alpha\left(T\left(P_{n}\right)\right)=\left\lfloor\frac{2\left|V\left(T\left(P_{n}\right)\right)\right|}{3}\right\rfloor, \quad \beta\left(T\left(P_{n}\right)\right)=\left\lceil\frac{\left|V\left(T\left(P_{n}\right)\right)\right|}{3}\right\rceil, \\
\sigma\left(T\left(P_{n}\right)\right)= \begin{cases}\frac{\left|V\left(T\left(P_{n}\right)\right)\right|}{5}, & \text { если }\left|V\left(T\left(P_{n}\right)\right)\right| \bmod 5=0, \\
\left.\mid \frac{\left|V\left(T\left(P_{n}\right)\right)\right|}{5}\right\rfloor+1 & \text { в противном случае. }\end{cases}
\end{gathered}
$$

Теорема 3.2. Пусть $C_{n}-$ иикл с $n$ вериинами. Тогда

$$
\begin{gathered}
k\left(T\left(C_{n}\right)\right)=4, \quad \alpha\left(T\left(C_{n}\right)\right)=\left\lceil\frac{2\left|V\left(T\left(C_{n}\right)\right)\right|}{3}\right\rceil, \quad \beta\left(T\left(C_{n}\right)\right)=\left\lfloor\frac{\left|V\left(T\left(C_{n}\right)\right)\right|}{3}\right\rfloor, \\
\sigma\left(T\left(C_{n}\right)\right)= \begin{cases}\frac{\left|V\left(T\left(C_{n}\right)\right)\right|}{5}, & \text { если }\left|V\left(T\left(C_{n}\right)\right)\right| \bmod 5=0, \\
\left.\mid \frac{\left|V\left(T\left(C_{n}\right)\right)\right|}{5}\right\rfloor+1 & \text { в противном случае. }\end{cases}
\end{gathered}
$$

ТЕОрема 3.3. Пусть $K_{n}-$ полный граф с п вериинами. Тогда

$$
\begin{gathered}
k\left(T\left(K_{n}\right)\right)=2(n-1), \quad \alpha\left(T\left(K_{n}\right)\right)=\left\lceil\frac{\left|V\left(T\left(K_{n}\right)\right)\right|(n-1)}{n}\right\rceil, \\
\beta\left(T\left(K_{n}\right)\right)=\left\lfloor\frac{\left|V\left(T\left(K_{n}\right)\right)\right|}{n}\right\rfloor, \quad \sigma\left(T\left(K_{n}\right)\right)=\left\lceil\frac{n}{2}\right\rceil .
\end{gathered}
$$

ТеОремА 3.4. Пусть $G$ - связный граф с $n$ вериинами $и \alpha(G)=1$. Тогда $\sigma(T(G))=1, k(T(G))=2 u \alpha(T(G))=n$.

ДокАЗАТЕльство. Если $\alpha(G)=1$, то в $G$ есть остовная звезда. Это означает, что и в тотальном графе графа $G$ есть остовная звезда. Отсюда $\sigma(T(G))=1, k(T(G))=2$ и $\alpha(T(G))=n$.

Теорема 3.5. Пусть $S_{1, n}-$ звезда с $n+1$ вериинами. Тогда $k\left(T\left(S_{1, n}\right)\right)=2$, $\alpha\left(T\left(S_{1, n}\right)\right)=n+1, \beta\left(T\left(S_{1, n}\right)\right)=n u \sigma\left(T\left(S_{1, n}\right)\right)=1$.

Теорема 3.6. Пусть $W_{1, n}-$ колесо с $n+1$ вериинами. Тогда

$$
\begin{gathered}
k\left(T\left(W_{1, n}\right)\right)=6, \quad \alpha\left(T\left(W_{1, n}\right)\right)=\left\lceil\frac{3\left|V\left(T\left(W_{1, n}\right)\right)\right|}{4}\right\rceil, \\
\beta\left(T\left(W_{1, n}\right)\right)=\left\lfloor\frac{\left|V\left(T\left(W_{1, n}\right)\right)\right|}{4}\right\rfloor, \quad \sigma\left(T\left(W_{1, n}\right)\right)=1+\left\lceil\frac{n}{3}\right\rceil .
\end{gathered}
$$

ТЕорема 3.7 [2]. Для любого графа $G$ справедливо неравенство

$$
k(G) \leqslant k_{1}(G) \leqslant \delta(G) .
$$


ТЕОРема 3.8. Для любого тотального графа $T(G)$ имеем

$$
\begin{array}{ll}
k(T(G))=2, & k_{i}(G)=1, \\
k(T(G)) \geqslant 2 k(G), & k_{i}(G)>1 .
\end{array}
$$

ДокАЗАТЕЛЬСтво. Доказательство теоремы состоит из двух частей.

Случай 1. Если $k_{1}(G)=1$, то $k(G)=1$. Предположим, что граф $G$ распадается при удалении ребра $\left\{e_{1}\right\}$. Из определения тотального графа вытекает, что этому ребру соответствует вершина в тотальном графе $T(G)$. Поскольку при удалении этого ребра граф $G$ распадается, по меньшей мере, на две компоненты, при удалении вершины $e_{1}$ в $T(G)$ и одной из вершин $u$ или $v$, каждая из которых является концом ребра $e_{1}$ в $G$, граф $T(G)$ тоже распадается. Поэтому

$$
k(T(G))=\min \left\{\left\{e_{1}, u\right\}\right\} \quad \text { или } \min \left\{\left\{e_{1}, v\right\}\right\}=2 .
$$

Случай 2. Вершины тотального графа $T(G)$ разбиваются на две части. Всевершины первой части являются вершинами графа $G$, а вершины из второй части соответствуют его ребрам. Степени вершин из первой части в два раза больше их степеней в $G$, поэтому $\delta_{1 \text { part }}(T(G))=2 \delta(G)$. Минимальная степень вершины из второй части не превосходит четырех, $\delta_{2 \text {. part }}(T(G)) \leqslant 4$. Поэтому в данном случае связность графа имеет вид

$$
k(T(G))=\min \left\{\delta_{1 . p a r t}(T(G)), \delta_{2 . \text { part }}(T(G))\right\}
$$

Если $k(T(G))=\delta_{1 . \text { part }}(T(G))$, то $k(T(G))=\delta_{1 . \text { part }}(T(G))=2 \delta(G) \geqslant 2 k(G)$ по теореме 3.7. Поэтому

$$
k(T(G)) \geqslant 2 k(G)
$$

Если $k(T(G))=\delta_{2 \text {. part }}(T(G))$, то $k(T(G))=\delta_{2 . \text { part }}(T(G)) \leqslant 4$. Поэтому

$$
k(T(G)) \leqslant 4
$$

Из (1) и (2) получаем

$$
2 k(G) \leqslant k(T(G)) \leqslant 4 .
$$

Совокупность утверждения случаев 1 и 2 завершает доказательство теоремы.

4. Целостность тотальных графов, связанная с некоторыми их характеристиками. В этом разделе мы приводим теоремы о целостности тотальных графов, которые соотносят друг с другом их связности, числа покрытия, индексы независимости и доминирования.

ТеОрема 4.1. Пусть $G-$ связный граф и пусть $\alpha(G)=1 ; \operatorname{moгда~} I(T(G))=n+2$. 
ДоКАЗАТЕЛЬСТво. Если $\alpha(G)=1$, то $G$ представляет собой звезду $S_{1, n}$ с $n+1$ вершинами. Кроме того, в графе $T(G)$ есть остовная звезда. Возможны три случая.

Случай 1. Пусть удаляемое множество $S$ - минимальное покрьвающее множество вершин графа $T(G)$. Тогда $m(T(G)-S)=1$. Поэтому $I(T(G))=\alpha(G)+1$. Из теоремы 3.4 вытекает, что $\alpha(T(G))=n+1$. Поэтому $I(T(G))=n+2$.

Случай 2. Пусть $S$ состоит из минимального покрьвающего подмножества графа $T(G)$ и некоторых других вершин. Тогда $|S| \geqslant \alpha(T(G))+1$. Поэтому $m(T(G)-S)=1$. Таким образом, $\min \{|S|+m(T(G)-S)\} \geqslant n+3$.

Случай 3 . Пусть $S$ является собственным подмножеством минимального покрьвающего множества графа $T(G)$. Тогда $|S|<\alpha(T(G))$. Одна из вершин минимального покрывающего множества графа $T(G)$ образует минимальное покрывающее множество звезды. Если мы удалим эту вершину из графа $T(G)$, то получим полньй граф, в котором каждая вершина соединена с каждой. Поэтому $|S|<\alpha(T(G))-p, p=1,2, \ldots$, $\alpha(T(G))-1$ и $m(T(G)-S)=2 p$. Значит,

$$
I(T(G))=\min \{|S|+m(T(G)-S)\}=\min \{n+1+p\}=n+1+p
$$

В силу неравенства $1 \leqslant p \leqslant \alpha(T(G))-1$ имеем $I(T(G)) \geqslant n+2$. Воспользовавшись результатами случаев $1-3$, получаем

$$
I(T(G))=\min \{n+2, n+3, n+2\}=n+2 .
$$

ТЕОремА 4.2. Пусть $G-$ связный граф с п вериинами $(n>4) ;$ тогда

$$
\beta(T(G))+k(T(G)) \leqslant I(T(G)) \leqslant \alpha(T(G))+1 .
$$

ДокАЗАТЕЛьство. Для доказательства рассмотрим два случая.

Случай 1 . Если множество удаляемых вершин $S$ - максимальное независимое подмножество в $T(G)$, то после удаления получаем $m(T(G)-S) \geqslant k(G)$. Поэтому

$$
I(T(G))=\min \{|S|+m(T(G)-S)\} \geqslant \min \{\beta(T(G))+k(T(G))\} \geqslant \beta(T(G))+k(T(G))
$$

и

$$
I(T(G)) \geqslant \beta(T(G))+k(T(G)) .
$$

Случай 2. Если мы хотим, чтобы максимальные компоненты имели порядок один, то число удаляемых вершин не меньше $|S| \leqslant \alpha(T(G))$. Поэтому

$$
I(T(G))=\min \{|S|+m(T(G)-S)\} \leqslant \min \{\alpha(T(G))+1\} \leqslant \alpha(T(G))+1 .
$$

В силу результатов случаев 1 и 2 имеем

$$
\beta(T(G))+k(T(G)) \leqslant I(T(G)) \leqslant \alpha(T(G))+1
$$

ТЕОремА 4.3. Если $T$ - дерево с п вериинами и $\alpha(T)=2$, то $\alpha(T(T)) \geqslant n$. 
ДокАЗАТЕЛЬСтво. Если $T$ - дерево с $n$ вершинами и $\alpha(T)=2$, то число ребер в $T$ равно $n-1$. Поэтому в тотальном графе $T(T)$ имеется $2 n-1$ вершин.

Минимальное покрьвающее подмножество тотального графа является объединением минимального покрывающего подмножества вершин графа $T$ и вершин, отвечающих его ребрам. Однако одна из вершин в $T(T)$, соответствующая ребру в $T$, примыкает к двум вершинам из минимального покрьвающего подмножества графа $T$ и, значит, не принадлежит минимальному покрывающему множеству графа $T(T)$. Поэтому

$$
\alpha(T(T)) \geqslant \alpha(T)+E(T)-1 \geqslant 2+n-1-1 \geqslant n .
$$

Tеорема 4.4. Если $T$ - дерево с п вериинами и $\alpha(T)=2$, то $I(T(T))=n+1$.

ДокАЗАТЕльство. Для вершины $v$ графа $G$ еезамкнутая окрестность определяется как объединение $\{v \cup N(v)\}$, где через $N(v)$ обозначено множество вершин, примыкающих к $v$. Если множество удаляемых вершин $S$ является минимальным покрывающим множеством дерева $T$, то $|S|=\alpha(T)=2$ и $m(T-S)=1$. Поэтому $\{S \cup N(S)\}=V(T)$. Все вершины графа $T(T)$ примыкают к вершинам из минимального покрьвающего множества дерева $T$. Кроме того, каждая из вершин графа $T$ примыкает по крайней мере к одной из вершин графа $T(T)$. Если $\alpha(T)=2$, то имеет место один из двух случаев.

Случай 1. Пусть множество удаляемых вершин $S$ графа $T(T)$ является его минимальным покрытием. Тогда $m(T(T)-S)=1$. Поэтому

$$
I(T(T))=\min \{|S|+m(T(T)-S)\}=\min \{\alpha(T(T))+1\} .
$$

Из теоремы 4.3 вытекает, что $I(T(T)) \geqslant \min \{n+1\}=n+1$.

Случай 2. Пусть $S$ является собственным подмножеством в минимальном покрытии графа $T(G)$. Тогда $|S|<\alpha(T(T))$, т.е. $|S|=\alpha(T(T))-p, p=1,2, \ldots, \alpha(T(T))-1$. Поэтому $m(T(T)-S) \geqslant p+2$, откуда

$$
\begin{aligned}
I(T(T)) & =\min \{|S|+m(T(T)-S)\} \geqslant \min \{\alpha(T(T))-p+p+2\} \\
& =\min \{\alpha(T(T))+2\} .
\end{aligned}
$$

Из теоремы 4.3 вытекает, что $I(T(T)) \geqslant \min \{n+2\}=n+2$.

Результаты случаев 1 и 2 дают $I(T(T))=\{n+1, n+2\}=n+1$.

Теорема 4.5. Для всех регулярных графов, если $\sigma(G)=\beta(G), \alpha(G)=k(G)$, то

$$
I(G) \geqslant k(T(G))+\sigma(T(G)) .
$$

ДокАЗАТЕЛЬСтво. Граф $G$ назьвается регулярным степени $r$ (или $r$-регулярным), если степень каждой его вершины равна $r$. Для таких графов $\delta(G)=\Delta(G)=k(G)=r$. В частности, если $G$ регулярный и $\sigma(G)=\beta(G), \alpha(G)=k(G)$, то из результата 2.7 вытекает, что

$$
I(G)=k(G)+1=r+1 .
$$

Тотальньй граф графа, обладающего таким свойством, также является регулярным. Поэтому

$$
\delta(T(G))=\Delta(T(G))=k(T(G))=2 r .
$$


Для такого тотального графа $\sigma(T(G))=\beta(T(G))$ и $k(T(G)) \neq \alpha(T(G))$. Пусть $S-I$-множество графа $T(G)$. В силу результата 2.8 имеем $|S| \geqslant k(T(G))$ и $m(T(G)-S) \geqslant(T(G))$. Поэтому

$$
\begin{aligned}
I(T(G)) & =\min \{|S|+m(T(G)-S)\} \\
& \geqslant \min \{k(T(G))+(T(G))\} \geqslant k(T(G))+(T(G)) .
\end{aligned}
$$

5. Заключение. Вычисление целостности графа $N P$-полная задача. Однако для некоторых классов графов целостность можно записать явной формулой. В этих случаях ее можно выразить через другие характеристики графов. Тотальные графы $T(G)$ строятся по отношениям примыканий в графах.

В этой статье мы рассматриваем тотальный граф $T(G)$ графа $G$. Ясно, что как порядок, так и связность графа $T(G)$, больше, чем у $G$. Мы доказываем теоремы, выражающие целостность тотального графа через некоторые его характеристики. При конструировании коммуникационных сетей с заданными характеристиками можно воспользоваться таблище 1.

ТАБлица 1. Целостность некоторых графов

\begin{tabular}{|c|c|c|c|c|}
\hline Характеристики & $\begin{array}{c}N \text { (число } \\
\text { вершин в } G)\end{array}$ & Граф & $I(G)$ & $I(T(G))$ \\
\hline \hline$\alpha(G)=1$ & 6 & $S_{1,5}$ & 2 & 7 \\
& 3 & $P_{3}$ & 2 & 4 \\
\hline$\alpha(G)=2$ & 7 & $T_{7}$ & 3 & 8 \\
$(G-$ дерево) & 5 & $P_{5}$ & 3 & 6 \\
\hline$\sigma(G)=\beta(G)$ и & 4 & $K_{4}$ & 4 & 9 \\
$\alpha(G)=k(G)$ & & $C_{4}$ & 3 & 6 \\
\hline
\end{tabular}

Из этой таблицы видно, что целостность тотальных графов превьшает целостность всех остальных, что и позволяет нам предпочесть тотальные графы.

\section{СПИСОК ЦИТИРОВАННОЙ ЛИТЕРАТУРЫ}

[1] Kumar V., Grama A., Gupta A., Karpis G. Introduction To Parallel Computing. California: The Benjamin Cummings Publ., 1995.

[2] Lesniak L., Chartrand G. Graphs and Digraphs. California: Wadsworth \& Brooks, 1986.

[3] Cozzens M. B., Wu S. Y. Edge neighbor-integrity of trees // Austral. J. Combinatorics. 1994. V. 10. P. 163-174.

[4] Cozzens M. B., Yu. S. Y. Vertex neighbor-integrity of trees // Ars. Combinatorial. 1996. V. 43. P. $169-180$.

[5] Chvatal V. Tough graphs and Hamiltonian circuits // Discrete Math. 1973. V. 5. P. 215-218.

[6] Dündar P. The tenacity of hypercube graphs and its some compounds // Neural Networks World. 1999. V. 3/99. P. 225-234.

[7] Düdar P. Stability measures of some static interconnection networks // Intern. J. Comput. Math. 2001. V. 76. №4. P. 455-462.

[8] Dündar P. Accessibility number and the neighbour-integrity of generalised Petersen graphs // Neural Network World. 2001. V. 2. P. 167-174.

[9] Dündar P., Ozan A. Stability measures of torus and mesh graphs // Intern. J. Math. Algorithms. 2002 (submitted). 
[10] Dündar P., Ozan A. The neighbour-integrity of sequential joined graphs // Intern. J. Comput. Math. 2000. V. 74. P. 45-52.

[11] Dündar P. The neighbour-integrity of Boolean graphs and its compounds // Intern. J. Comput. Math. 1999. V. 72. P. 441-447.

[12] Dündar P. New notions in network reliability: stability numbers of sequential joined graphs // Neural Network World. 1999. V. 5. P. 403-411.

[13] Dündar P. The stability measures of static interconnection networks and binary trees // Proceeding of the Third International Symposium on Computer Networks. Izmir, 1998. P. 240-245.

[14] Dündar P., Ten O. A new parameter for network stability: the neighbour-tenacity // Proceedings of the 14th Intern. Symposium on Comput. and Information Sciences. Kusadasi, 1999. P. 1016-1018.

[15] Dündar P., Öztürk N. The neighbour-integrity of recursive graphs // Proceedings of the 2nd Intern. Symposium on Math. and Computational Applications. Baku, 1999. P. 443-447.

[16] Bagga K. S., Beineke L. W. Survey of integrity // Discrete Appl. Math. 1992. V. 37/38. P. 13-28.

[17] Barefoot C. A., Entringer R. Vulnerability in graphs-a comparative survey // J. Combin. Math. Combin. 1989. P. 113-122.

[18] Beinike L. W., Goddard W., Hamburger P., Kleitman D. J., Lipman M. J., Pippert R. E. The integrity of the cube is small // J. Combin. Math. Combin. Comput. 1991. V. 9. P. 191-193.

[19] Goddard W., Swart H. C. Integrity in graphs: bounds and basics // J. Combin. Math. Combin. Comput. 1990. V. 7. P. 139-151.

[20] Bagga K. S., Beineke L. W., Lipman M. J., Pippert R. E. Edge-integrity: a survey // Discrete Math. 1994. V. 124. P. 3-12.

[21] Bagga K.S., Beineke L. W., Lipman M. J., Pippert R. E. On the edge-integrity of graphs // Congres. Numer. 1987. V. 60. P. 141-144.

[22] Bagga K.S., Beineke L. W., Lipman M. J., Pippert R. E., Sedlmeyer R. L. A good algorithm for the computation of the edge-integrity of trees // Congres. Numer. 1988. V. 67. P. 225-232.

[23] Harary F. Graph Theory. California: Addison-Wesley Publ., 1971.

University of Ege, Турция

Поступило

E-mail: dundar@sci.ege.edu.tr, aozan@sci.ege.edu.tr

02.04 .2003 